\title{
Bowel Preparation for a Better Colonoscopy Using Polyethylene Glycol or C-lax: A Double Blind Randomized Clinical Trial
}

\author{
Rose Khorasanynejad ${ }^{1}$, Alireza Norouzi ${ }^{2, *}$, Gholamreza Roshandel ${ }^{3}$, Sima Besharat ${ }^{4}$
}

\section{ABSTRACT}

1. Assistant of cardiology, Department of Cardiology, Mashhad University of Medical Sciences, Mashhad, Iran

2. Gastroentrologist, Golestan Research Center of Gastroenterology and Hepatology, Golestan University of Medical Sciences, Gorgan, Iran

3. Epidemiologist, Golestan Research Center of Gastroenterology and Hepatology, Golestan University of Medical Sciences, Gorgan, Iran

4. PhD of Biomedicine, Golestan Research Center of Gastroenterology and Hepatology, Golestan University of Medical Sciences, Gorgan, Iran

\section{* Corresponding Author:}

Alireza Norouzi, MD

Golestan Research Center of Gastroenterology and Hepatology, Third floor, Research Centers' Complex, Sayyade-Shirazi Hospital, Sayyad-e-Shirazi Boulevard, Gorgan, Iran Telefax: + 981732251910 Email: norouzi54@gmail.com

Received: 16 May 2017

Accepted: 11 Aug. 2017

\section{BACKGROUND}

Ideal bowel preparation regimen for a suitable colonoscopy should be safe, and well tolerated, and should rapidly clear gastrointestinal tract. Soluble polyethylene glycol (PEG) is the most common cleansing drug and Senna or C-Lax (Cassia angustifolia Vahl) is an alternative herbal one. This study was designed to compare the efficacy of PEG and C-lax in bowel preparation.

\section{METHODS}

In this randomized double blind trial (registry number in IRCT.ir: IRCT201601161264N7), 320 patients were randomly assigned in PEG or C-lax groups. PEG solution was prepared from $5 \times 70$ gr sachets in $20 \times 250 \mathrm{cc}$ water $(250 \mathrm{ml}$ every 15 minutes $)$, prescribed $24 \mathrm{~h}$ before the colonoscopy. In the other group $3 \times 60 \mathrm{ml} \mathrm{C}$-lax syrup glasses (each containing $90 \mathrm{mg}$ senozid B) was given in two divided doses ( 1.5 glasses of $250 \mathrm{cc}$ every 12 hours), $24 \mathrm{~h}$ before the colonoscopy. Ottawa score was used to evaluate the quality of bowel preparation. Chi-square test, Student $t$ test, MannWhitney test and multivariate analysis were used to analyze the data.

\section{RESULTS}

Of these patients with the mean (SD) age of 50 (15.16) years, $162(50.8 \%)$ were men. Mean (SD) Ottawa score was $2.57(0.2)$ and $3.15(0.31)$ in the PEG and C-lax group, respectively (p value $=0.81)$. Multivariate analysis showed that less opium consumption $(\mathrm{p}<0.001)$ and higher educational level $(\mathrm{p}=0.005)$ were associated with better bowel preparation.

\section{CONCLUSION}

C-Lax is non-inferior to PEG solution in cleansing colon. The quality of bowel preparation was lower in opium consumers and better in those with higher educational level.

\section{KEYWORDS:}

Bowel preparation, Polyethylene glycol, C-Lax, Double blind clinical trial, Ottawa score

Please cite this paper as:

Khorasanynejad R, Norouzi AR, Roshandel GR, Besharat S. Bowel Preparation for a Better Colonoscopy Using Polyethylene Glycol or C-lax: A Double Blind Randomized Clinical Trial. Middle East J Dig Dis 2017;9:212-217. doi: 10.15171/mejdd.2017.76.

\section{INTRODUCTION}

Colonoscopic examination necessitates a well prepared fecal-matter-free bowel for a diagnostic and therapeutic approach. ${ }^{1}$ A good colonoscopy may have three main characteristics: a skilled gastroenterologist, a cooperative patient, and a glossy clean bowel. ${ }^{2}$ Bowel preparation most commonly involves oral ingestion of a large volume of a cathartic agent with laxative properties over a defined period of time. ${ }^{1,3}$

Administering a good tasty tolerable oral agent to patients willing to undergo a colonoscopic examination may seem no big deal to clinicians but is a key factor for patients to decide whether to avoid or accept the procedure. ${ }^{4}$ Efficacy, tolerance, and safety are among the most important factors that a 
cleansing agent should have when chosen. ${ }^{4}$

Inadequate bowel cleanliness has been reported in one third of patients who have been candidate for colonoscopy ${ }^{5}$, leading to a higher rate of repeat colonoscopy, increased rate of complications, and a longer duration of the procedure. ${ }^{4,6,7}$

There are several factors affecting bowel preparation such as face-to-face patients' education, patients' general health condition like elderly, or childhood, inflammatory bowel disease in active phase, or concurrent renal failure, heart failure, acute gastrointestinal bleeding, and urgent colonoscopy. Such factors can affect the ability of patients to tolerate and adhere to the preparation regimen. ${ }^{1,4}$

Three types of bowel preparation agents are available so far: a- polyethylene glycol (PEG) solutions, b-osmotic agents, such as sodium phosphate, magnesium citrate, lactulose, and mannitol, and c- stimulants, such as castor oil, Senna, sodium picosulfate, and bisacodyl. ${ }^{2}$

PEG is a balanced isotonic oral, non-digestible, and non-absorbable solution, of which usually 4 liters are administrated for bowel preparation. In some patients, the unpleasant taste and smell and drinking a large volume in short time intervals could not be well tolerated. ${ }^{1,4}$

Stimulants affect the epithelial transport of water and electrolytes and stimulate the intestinal motility. They are cheaper, safe, and easy to ingest. Senna (Cassia angustifolia Vahl, Leguminosae, Indian Senna, Tinnevelly Senna) is a laxative from this group. ${ }^{8}$

In the present study, we compared the quality of bowel preparation with either PEG solution or Senna in two groups of patients who were candidate for colonoscopy in our academic center, in northeast Iran.

\section{MATERIALS AND METHODS}

\section{Randomization}

In this double blind randomized clinical trial, 320 eligible patients were recruited from the candidates with any indications of colonoscopy such as screening, polyp surveillance, etc who were referred to our academic hospital in Gorgan city, northeast Iran. All the patients were randomly allocated in either PEG $(\mathrm{N}=160)$ or $\mathrm{C}$ lax $(\mathrm{N}=160)$ group by the endoscopy ward technician. Neither the gastroenterologist nor the one who evaluated the Ottawa score was aware of the group randomization. Age and sex were matched in both groups using frequency matching method.

A soft clear liquid regimen without any solid residues (bread, rice, vegetables or fruits) was administered to all the patients the day before the procedure. In PEG group, $5 \mathrm{~L}$ of PEG fluid was administered (sachets of PEG powder were rinsed in water, each sachet contains 70 grams of PEG rinsed in 4 glasses of water), and the patients were asked to drink $250 \mathrm{~mL}$ of the solution every 15 minutes starting 24 hours before the procedure.

In C-lax group, three bottles of C-lax syrup (each 60 $\mathrm{mL}$ bottle contains $90 \mathrm{mg}$ Senozoids) in two split doses beginning from 24 hours before the procedure ( 1.5 bottles at 9 am and 1.5 bottles at $5 \mathrm{pm}$ the day before colonoscopy) were prescribed.

Cases were suggested to drink lots of liquids. More liquid food and beverage were suggested for those with long-lasting constipation. A written permission from a cardiologist was also necessary for those with a history of heart disease (in addition to self-informed consent).

Exclusion criteria were as following: age less than 18 years, a history of colectomy, any contraindication for colonoscopy, severe mental disease, pregnancy, lactation, and patients' not willing to participate.

A single gastroenterologist (principle investigator of the study) performed all colonoscopies and scored the bowel preparation using Ottawa bowel preparation quality scale (table 1). This system evaluates the bowel cleansing and fluid volume separately for the right colon (secum and ascending colon), mid colon (descending and transverse colon) and rectosigmoid colon. Each part of the colon would be scored from 0 to 4 and the liquid is scored from 0 to 2 ( 0 : minimal liquid, 1: moderate, and 2: lots of liquid). Total score would be from 0 to 14 (solid stool in entire colon and lots of liquid, no preparation). Lower score means the better preparation and 14 means the worst.

\section{Outcome measure}

The quality of bowel preparation by either methods (PEG or C-Lax) measured by Ottawa score was considered as the primary outcome. Adequate bowel preparation was defined as a total Ottawa score of $\leq 4$, while inadequate bowel preparation was defined as a score of more than 5 , based on the previous studies. ${ }^{7}$ 
Table 1: Comparing baseline characteristics of the two studied groups receiving either PEG or C-Lax

\begin{tabular}{|c|c|c|c|}
\hline \multirow[b]{2}{*}{ Variables } & \multicolumn{2}{|c|}{ Intervention group } & \multirow[b]{2}{*}{ Total } \\
\hline & $\begin{array}{c}\text { PEG } \\
(\mathrm{N}=160)\end{array}$ & $\begin{array}{c}\text { C-Lax } \\
(N=151)\end{array}$ & \\
\hline \multicolumn{4}{|l|}{ Sex } \\
\hline Male/ Female & 83.77 & 75.76 & 0.619 \\
\hline \multicolumn{4}{|l|}{ Education level } \\
\hline Illiterate & $50(30.7)^{*}$ & $47(30.1)$ & \multirow{3}{*}{0.994} \\
\hline High school & 65 (39.9) & $63(40.4)$ & \\
\hline College & $48(29.4)$ & $46(29.5)$ & \\
\hline \multicolumn{4}{|l|}{ Ethnicity } \\
\hline Fars & $10(6.1)$ & $7(4.5)$ & \multirow{3}{*}{0.691} \\
\hline Turkmen & $122(74.8)$ & $114(73.5)$ & \\
\hline Other & $31(19)$ & $34(21.9)$ & \\
\hline \multicolumn{4}{|l|}{ Smoking history } \\
\hline No & $138(88.5)$ & $144(94.1)$ & \multirow{2}{*}{0.078} \\
\hline Yes & $18(11.5)$ & $9(5.9)$ & \\
\hline \multicolumn{4}{|c|}{ Opium consumption } \\
\hline No & $132(83)$ & $128(83.7)$ & \multirow{2}{*}{0.879} \\
\hline Yes & $27(17)$ & $25(16.3)$ & \\
\hline
\end{tabular}

\section{Ethical considerations}

The study protocol was approved by the local Ethics Committee of Golestan University of Medical Sciences (Reference number: 32189210226). This clinical trial was registered in Iranian registry of clinical trials (www. IRCT.ir) and assigned a code as: IRCT201601161264N7. Informed consent was obtained from all the participants after verbal consent and explaining the study protocol by the principle investigator.

\section{Statistical analysis}

SPSS software version 16 was used for analysis. Chi square test was used to analyze qualitative variables and $t$ test was used for quantitative ones after testing for the normality of the variables by Kolmogorov-Smirnov test. $p$ value less than 0.05 was considered as statistically significant.

\section{RESULTS}

In this randomized clinical trial, 320 eligible cases were randomized into either PEG or C-Lax group. Nine cases from C-Lax group did not present in the scheduled date, despite phone calls and follow-ups and were excluded from the study. So, all analyses were done on 151 cases in C-lax group and 160 cases in PEG group.

The mean (SD) age of the studied patients was 50 (15.2) years. $158(51 \%)$ patients were male and 153 $(49 \%)$ were female. There were no significant differences between the groups receiving PEG or C-Lax regarding the demographic variables (table 1).

Mean (SD) Ottawa score was not significantly different between PEG and C-Lax groups, 2.6 (2.5) versus 3.15 (3.75), respectively $(p=0.110)$.

As shown in table 2, in PEG group there was a significant higher Ottawa score in illiterates and opium consumers $(p<0.005)$. And in C-Lax group, elder patients ( $\geq 51$ years), illiterate cases, ethnic groups other than Fars and Turkmen and opium consumers had significantly higher Ottawa score $(p<0.005$, table 2).

Multivariate analysis showed that opium consumption and education level were the strongest variables related to the bowel preparation. Opium increased the Ottawa score (worse bowel preparation) but higher education level decreased the Ottawa score (better bowel preparation, table 3).

As shown in table 4, mean (SE) Ottawa score was not significantly different in different segments of the colon in both groups, although the score was lower for the entire colon in PEG group compared with the C-lax group (table 4).

\section{DISCUSSION}

In this randomized clinical trial, we found no significant differences in bowel preparation scores between those cases taking PEG or C-Lax. However, opium consumers and those with lower formal educational level showed significant lower bowel preparation quality in both groups.

According to the literature, some studies reported the superiority and effectiveness of PEG solution ${ }^{9,10}$ and some showed equal ${ }^{11-13}$ or inferior effects ${ }^{14,15}$ for PEG group compared with Sennosides.

Radaelli and colleagues compared the efficacy and overall quality of colon cleansing of 24 tablets of $12-\mathrm{mg}$ Senna (divided into two doses at 1 p.m. and 9 p.m. [Senna group, $n=191]$ ) with standard 4-L Polyethylene glycol-electrolyte solution (PEG-ES group, $\mathrm{n}=92$ ). Consumption of Senna revealed significantly better quality 
Table 2: Comparing mean (SE) Ottawa score regards to the baseline characteristics of the studied population

\begin{tabular}{|c|c|c|c|c|c|}
\hline Variables & & PEG $(\mathrm{N}=160)$ & $p$-value & C-Lax $(N=151)$ & $p$-value \\
\hline \multirow[t]{2}{*}{ Sex } & Male & $2.45(0.24)$ & \multirow{2}{*}{0.78} & $3.39(0.41)$ & \multirow{2}{*}{0.12} \\
\hline & Female & $2.71(0.31)$ & & $2.91(0.44)$ & \\
\hline \multirow[t]{2}{*}{ Age group } & $<51$ & $2.16(0.22)$ & \multirow{2}{*}{0.07} & $2.66(0.40)$ & \multirow{2}{*}{0.03} \\
\hline & $\geq 51$ & $2.95(0.3)$ & & $3.65(0.45)$ & \\
\hline \multirow[t]{3}{*}{ Education level } & Illiterate & $3.32(0.41)$ & \multirow{3}{*}{0.002} & $4.82(0.75)$ & \multirow{3}{*}{0.004} \\
\hline & High school & $2.66(0.3)$ & & $2.91(0.39)$ & \\
\hline & College & $1.68(0.24)$ & & $1.84(0.34)$ & \\
\hline \multirow[t]{3}{*}{ Ethnicity } & Fars & $2.17(0.8)$ & \multirow{3}{*}{0.47} & $2.75(0.52)$ & \multirow{3}{*}{0.004} \\
\hline & Turkmen & $2.39(0.19)$ & & $2.60(0.31)$ & \\
\hline & Others & $3.4(6.4)$ & & $4.99(0.81)$ & \\
\hline \multirow[t]{2}{*}{ Cigarette smoking } & Yes & $3.03(0.46)$ & \multirow{2}{*}{0.12} & $4.89(1.72)$ & \multirow{2}{*}{0.14} \\
\hline & No & $2.53(0.22)$ & & $2.97(0.30)$ & \\
\hline \multirow[t]{2}{*}{ Opium } & Yes & $4.89(0.71)$ & \multirow{2}{*}{0.001} & $5.73(1.01)$ & \multirow{2}{*}{0.001} \\
\hline & No & $2.10(0.16)$ & & $2.58(0.29)$ & \\
\hline
\end{tabular}

Table 3: Multivariate analysis of factors predicting the bowel preparation (Ottawa score)

\begin{tabular}{lcccc}
\hline Variables & Standardized Beta & Unstandardized Beta & $\mathbf{9 5 \%}$ Confidence Interval for B & $p$-value \\
\hline C-Lax or PEG & 0.08 & 0.52 & $-0.14-1.19$ & 0.124 \\
\hline Sex & -0.01 & -0.09 & $-0.80-0.61$ & 0.792 \\
\hline Age group & 0.03 & 0.19 & $-0.60-0.98$ & 0.638 \\
\hline Education & -0.17 & -0.73 & $-1.24-0.22$ & 0.005 \\
\hline Ethnicity & 0.10 & 0.70 & $-.01-1.42$ & 0.056 \\
\hline Co-morbidity & -0.03 & -0.20 & $-1.00-0.58$ & 0.609 \\
\hline Cigar & 0.01 & 0.15 & $-1.12-1.42$ & 0.817 \\
\hline Opium & 0.28 & 2.39 & $1.41-3.38$ & 0.000 \\
\hline
\end{tabular}

Table 4: Comparing mean (SE) Ottawa score of different part of colon in two groups of bowel preparation

\begin{tabular}{lccccc}
\hline & \multicolumn{5}{c}{ Mean (SE) of Ottawa score } \\
\cline { 2 - 6 } & Right colon & Mid colon & Rectosigmoid colon & Entire colon & Total score \\
\hline PEG $(\mathbf{N}=\mathbf{1 6 0})$ & $1.02(1.06)$ & $0.6(1.02)$ & $0.7(1)$ & $0.3(0.4)$ & $2.6(2.5)$ \\
\hline C-Lax $(\mathbf{N}=\mathbf{1 5 1})$ & $1.22(1.42)$ & $0.7(1.3)$ & $0.85(1.22)$ & $0.41(0.6)$ & $3.15(3.75)$ \\
\hline $\boldsymbol{P}$-value & 0.160 & 0.443 & 0.198 & 0.045 & 0.110 \\
\hline
\end{tabular}

of colon cleansing (90.6\% vs. $79.7 \%)$, overall tolerance of the preparation, and compliance $(p=0.003) .{ }^{15}$

Shavakhi and co-workers enrolled 322 patients randomized into two groups: Senna group (24 tablets of $11 \mathrm{mg}$ Senna in two divided doses 24 hours before colonoscopy) and PEG group (solution of four sachets in four liters of water the day before the procedure and the patients were asked to drink $250 \mathrm{ml}$ every 15 minutes).
The overall quality of colon cleansing was evaluated using the Aronchick scoring scale. Quality of colon cleansing, patients' tolerance, compliance, and the difficulty of the procedure was similar between the two groups $(p>0.05) .{ }^{13}$

Performance of colonoscopy in hospital inpatients, bowel habits tend to chronic constipation, opioid dependency, consumption of drugs such as tricyclic antidepres- 
sants (TCA), low socioeconomic status, being overweight or obese especially higher abdominal circumference, male sex, and starting the procedure later in a day are factors predicting a poor colonoscopy. ${ }^{5,16}$ Outpatient status has been also considered a difficult entity due to unawareness and poor adherence to the instructions. ${ }^{5}$

Type of cleansing agent, taking adequate water till the colonoscopy time, applying protocol, and the time interval between starting the preparation schedule and the colonoscopy procedure are the most important predictors of adequate bowel preparation. ${ }^{5,17}$ Different educational aids such as videos, booklets, questionnaires, telephone calls at the same day of colonoscopy or the short-message services, brief educational sessions and interactive systems have been applied to improve the bowel cleanliness, although inconsistent results have been achieved. $5,7,16,17$

Rosenfeld and colleagues in an interventional study on 38 inpatients, who were candidate for colonoscopy, randomized 16 cases to the intervention and 22 as controls. The patients in the intervention group received a brief counseling session in addition to written instructions outlining the methods and rationale for bowel preparation before colonoscopy. Results showed that a simple, inexpensive and safe method like counseling would significantly improve the outcome of colonoscopy. ${ }^{17}$ Prakash and colleagues in a series of outpatients referred for colonoscopy reported an improvement in the quality of bowel preparation scale (Ottawa score) when an additional educational video was added to the applied instruction. Although income level, education level or age had no significant correlation with Ottawa score. ${ }^{16}$ Forgetting the key components of the bowel preparation process urge the need to review the whole schedule using any educational facilities can be beneficial in answering the remained questions and empower patients' compliance with the bowel preparation. ${ }^{16}$

Furthermore, understanding the written instructions or counseling sessions are much better when patients have higher formal educational level (high school or more), however this may not be applicable for the visual educations like video tapes. Another interventional study showed that a short 10-minute physician-delivered patient education session along with the written instruction has better effect on the quality of bowel preparation. ${ }^{5}$
Applying a comfortable session to explain the rationale and importance of pre-colonoscopy preparation for patients would be of more importance especially when the educational level is low.

In the present study, we did not assess the effect of education on the quality of bowel preparation, but our results showed that higher educational level decreased the Ottawa score and provide a better bowel preparation.

\section{CONCLUSION}

It seems that PEG solutions and C-Lax syrup have similar effects in bowel preparation. But there are other factors resulting in a well prepared bowel such as the educational level and addiction to opioids. So, sociodemographic situation of the studied population would affect the outcome of even a well-designed colonoscopy. These demographic variables would be further investigated in future projects.

\section{ACKNOWLEDGEMENTS}

Authors tend to thank the research deputy of Golestan University of Medical Sciences for the financial support. This paper was extracted from a doctorate thesis dedicated to obtain an MD degree.

\section{CONFLICT OF INTEREST}

The authors declare no conflict of interest related to this work.

\section{REFERENCES}

1. Lim YJ, Hong SJ. What is the best strategy for successful bowel preparation under special conditions? World J Gastroenterol 2014;20:2741-45. doi: 10.3748/wjg.v20.i11.2741.

2. Park JB, Lee YK, Yang CH. The evolution of bowel preparation and new developments. Korean J Gastroenterol 2014;63:268-75. doi:10.4166/kjg.2014.63.5.268.

3. Toledo T, DiPalma J. Colon cleansing preparation for gastrointestinal procedures. Aliment Pharmacol Ther 2001;15:605-11. doi:10.1046/j.1365-2036.2001.00966.x

4. Parra-Blanco A, Ruiz A, Alvarez-Lobos M, Amorós A, Gana JC, Ibáñez P, et al. Achieving the best bowel preparation for colonoscopy. World J Gastroenterol 2014;20:17709-26. doi: 10.3748/wjg.v20.i47.17709.

5. Shieh TY, Chen MJ, Chang CW, Hung CY, Hu KC, Kuo $\mathrm{YC}$, et al. Effect of physician-delivered patient education on the quality of bowel preparation for screening colonoscopy. Gastroenterol Res Pract 2013;2013:570180. doi: 10.1155/2013/570180. 
6. Bechtold ML, Choudhary A. Bowel preparation prior to colonoscopy: a continual search for excellence. World $J$ Gastroenterol 2013;19:155-7. doi: 10.3748/wjg.v19.i2.155.

7. Park J, Kim TO, Lee NY, Kim H, Seo EH, Heo NY, et al. The Effectiveness of Short Message Service to Assure the Preparation-to-Colonoscopy Interval before Bowel Preparation for Colonoscopy. Gastroenterol Res Pract 2015;2015:628049. doi:10.1155/2015/628049.

8. Kositchaiwat S, Suwanthanmma W, Suvikapakornkul $\mathrm{R}$, Tiewthanom V, Rerkpatanakit P, Tinkornrusmee C. Comparative study of two bowel preparation regimens for colonoscopy: Senna tablets vs sodium phosphate solution. World J Gastroenterol 2006;12:5536-9. doi: 10.3748/wjg.v12.i34.5536.

9. Terry NA, Chen-Lim ML, Ely E, Jatla M, Ciavardone $\mathrm{D}$, Esch S, et al. Polyethylene glycol powder solution versus Senna for bowel preparation for colonoscopy in children. J Pediatr Gastroenterol Nutr 2013;56:215-9. doi:10.1097/MPG.0b013e3182633d0a.

10. Amato A, Radaelli F, Paggi S, Terruzzi V. Half doses of PEG-ES and Senna vs. high-dose Senna for bowel cleansing before colonoscopy: a randomized, investigator-blinded trial. Am J Gastroenterol 2010;105:675-81. doi:10.1038/ajg.2009.598.

11. Kierkus J, Horvath A, Szychta M, Woynarowski M, Wegner A, Wiernicka A, et al. High- versus low-volume polyethylene glycol plus laxative versus sennosides for colonoscopy preparation in children. $J$ Pediatr Gastroenterol Nutr 2013;57:230-5. doi: 10.1097/ MPG.0b013e3182950ef5.

12. Hookey LC, Depew WT, Vanner SJ. Combined low volume polyethylene glycol solution plus stimulant laxatives versus standard volume polyethylene glycol solution: a prospective, randomized study of colon cleansing before colonoscopy. Can J Gastroenterol 2006;20:101-5. doi:10.1155/2006/621367.

13. Shavakhi A, Kianinia M, Torabi G, Nemati A, Saeidian B, Hoseinzadeh M, et al. High dose Senna or Poly Ethylene Glycol (PEG) for elective colonoscopy preparation: a prospective randomized investigator-blinded clinical trial. J Res Med Sci 2011;16:149-55.

14. Farca BA, Fernández CE, Presenda MF. Comparative study of the use of sennoside A and B vs polyethylene glycol and electrolytes in anterograde preparation of the colon. Rev Gastroenterol Mex 1998;64:85-8.

15. Radaelli F, Meucci G, Imperiali G, Spinzi G, Strocchi E, Terruzzi V, et al. High-dose Senna compared with conventional PEG-ES lavage as bowel preparation for elective colonoscopy: a prospective, randomized, investigator-blinded trial High-Dose Senna for Colonoscopy Preparation. Am J Gastroenterol 2005;100:2674-80. doi:10.1111/j.1572-0241.2005.00335.x.

16. Prakash SR, Verma S, McGowan J, Smith BE, Shroff A, Gibson GH, et al. Improving the quality of colonoscopy bowel preparation using an educational video. Can J Gastroenterol Hepatol 2013;27:696-700. doi: $10.1155 / 2013 / 292636$.
17. Rosenfeld G, Krygier D, Enns RA, Singham J, Wiesinger $\mathrm{H}$, Bressler B. The impact of patient education on the quality of inpatient bowel preparation for colonoscopy. Can J Gastroenterol 2010;24:543-46. doi: $10.1155 / 2010 / 718628$. 